\title{
Driving Skills in HIV-Infected Patients Well Controlled with Antiretroviral Therapy
}

\author{
Carlos Tornero $^{1}$, Inmaculada Poquet ${ }^{1}$, Marine Bourguet ${ }^{1}$, Fernando Gomis-Pajares ${ }^{2 *}$ \\ ${ }^{1}$ Ana Ventura, Maricarmen Mafé, Internal Medicine Department, Hospital Francisco de Borja, Gandia, España; ${ }^{2}$ Preventive Service, \\ Hospital Francisco de Borja, Gandia, España. \\ Email: "tornero_car@gva.es, ctornero@ono.com
}

Received June $5^{\text {th }}$, 2012; revised July $2^{\text {nd }}$, 2012; accepted July $13^{\text {th }}, 2012$

\begin{abstract}
A study was made to determine whether HIV-infected patients with prolonged virological control suffer subclinical neurocognitive problems capable of interfering with driving skills, compared with the general population, and to explore the possible existence of differences between those treated with and without efavirenz. Material and Methods: We included 40 patients without history of neoplasm, psychiatric disorders or infections of the central nervous system associated or not to HIV, with stable and effective antiretroviral therapy during at least 48 months. Use was made of the ASDE DRIVER TEST N-845 standardized by the Spanish traffic authorities, and for which data corresponding to the Spanish general population were obtained from the manufacturer of the test battery. The Student t-test was used to compare the different variables with the population standards, and the comparison of proportions Z-statistic was used to determine the proportion of subjects above the accepted limit of normality cutoff point. These analyses were replicated for the two sub-samples (with or without efavirenz therapy), with a 95\% confidence level. The SPSS version 15 statistical package and Epidat 3.1 program were used. Results: The scores obtained in the HIV group were significantly poorer in the anticipation speed tests and in one of the multiple reactions test, though better results were obtained in the bimanual visual-motor coordination test. There were no differences in the percentages of patients with scores below the recommended limits. On comparing the treatment subgroups (efavirenz versus protease inhibitor), no differences were recorded in any of the study variables, and the differences with respect to the general population were the same as those described for the global group. Conclusions: Little differences were observed in driving skills in HIV well controlled HIV patients of minor clinical significance, and no differences were found in driving skills between the patients administered Efavirenz and those receiving protease inhibitor treatments.
\end{abstract}

Keywords: Driving Skills; Efavirenz; Neurocognitive Disorders

\section{Introduction}

Infection produced by the human immunodeficiency virus (HIV) has become a treatable chronic disease in which the current therapies aim to secure virological control and immunological recovery, but also to preserve patient quality of life with a view to allowing the return to normal social and occupational life.

The preferential treatment protocols often include efavirenz (EFV). This drug has effects upon the central nervous system (CNS) that are particularly intense in the first weeks of therapy, but which become attenuated over time. Nevertheless, some authors have reported sleep and mood disturbances, apathy and concentration difficulties in patients subjected to chronic treatment with EFV [1-3].

On the other hand, there is great interest in exploring the neurocognitive disorders experienced by HIV-in-

"Corresponding author. fected patients under conditions of virological control and immunological recovery. Subcortical and cortical disorders have been described in $30 \%-70 \%$ of all well controlled patients, mostly in the form of asymptomatic alterations or minor neurocognitive disorders. Such alterations have been associated to factors such as CD4 nadir, antiviral drug penetration of the CNS, or immune activetion $[4,5]$. Some studies have described increased patient driving risk as a result of these alterations [6,7].

The present study aims to determine whether HIV-infected patients with prolonged virological control suffer subclinical neurocognitive problems capable of interfering with driving skills, compared with the general population, and to explore the possible existence of differences between those treated with and without efavirenz.

\section{Material and Methods}

An analytical study was carried out, comparing a sample 
of HIV-infected patients with the data referred to the general population. Those patients meeting the following criteria were consecutively included until and estimated sample size of 40 was reached:

- A diagnosis of HIV infection.

- Stable and effective antiretroviral therapy (ART) maintaining viremias below the limit of detection for at least 48 months. Viral blips were not excluded, provided viremia returned to undetectable levels, with no appearance of resistances or need for modifications in ART.

- Maintenance during the mentioned time of the same treatment with EFV or with one of the protease inhibitors (PIs).

- Compilation of a full clinical history.

Patients with a history of neoplasms or infections of the CNS associated or not to HIV were excluded, as were patients suffering psychiatric disorders and subjected to treatments capable of interfering with the results (neuroleptics, benzodiazepines, hypnotics, or active drug abuse). Non-sedating antidepressants and stable maintenance therapy with methadone were allowed.

The exposure variables were assignation to the HIV infection group or the general population, while the outcome variables were those obtained after applying the psycho-technical ASDE DRIVER TEST N-845 standardized by the Spanish traffic authorities, and which comprises four groups of tests:

- Anticipation speed: Measures subject capacity to perceive speeds.

- Bimanual visual-motor coordination: Evaluates subject coordination of both hands simultaneously and independently.

- Multiple reaction times: Evaluates time and precision in responding to a stimulus of the four extremities.

- Concentration alteration and resistance to monotony test.

All tests were preceded by a learning phase to ensure motivation and understanding of the test. The data corresponding to the Spanish general population were obtained from the manufacturer of the test battery.

In addition, information was collected referred to educational level, the use of methadone, alcohol abuse, and CD4 current count and nadir.

Regarding the statistical analysis, we first made a descriptive study of the HIV-infected patient group-quantitative variables being reported as their central tendency (mean and median) and dispersion measures (standard deviation), and qualitative variables as absolute and relative (\%) frequencies. The Student t-test was used to compare the different variables with the population standards in the cases of quantitative variables (after confirming normal distribution). For each outcome variable we likewise used the Z-statistic to determine the proportion of subjects above the accepted limit of normality cutoff point. These analyses were applied to both subsamples (with or without EFV therapy). A 95\% confidence level was considered in all contrasts. Creation of the HIV-infected patient database and calculation of the descriptive parameters were based on the SPSS version 15 statistical package, while the Epidat 3.1 program was used for the bivariate analysis. A p $<0.05$ was considered of statistic significance.

\section{Results}

The baseline characteristics of the patients are shown in Table 1. Compared with the general population data, the

Table 1. Characteristics of the study patients. Categorical variables are expressed as n (\%), while quantitative variables are expressed as mean (standard deviation).

\begin{tabular}{cccc}
\hline & Total HIV $(\mathrm{N}=40)$ & HIV-efavirenz & HIV-No efavirenz (n=19) \\
\hline Male gender & $28(70.0)$ & $16(76.2)$ & $12(63.2)$ \\
Risk group & & & \\
Intravenous drug abuse & $18(45.0)$ & $6(28.6)$ & $12(63.2)$ \\
Heterosexual & $14(35.0)$ & $11(52.4)$ & $3(15.8)$ \\
Homosexual & $6(15.0)$ & $3(14.3)$ & $3(15.8)$ \\
Not known & $2(5.0)$ & $1(4.8)$ & $1(5.3)$ \\
Methadone treatment & $2(2.2)$ & $1(4.8)$ & $1(5.3)$ \\
Alcohol abuse & $3(2.2)$ & $1(4.8)$ & $2(10.5)$ \\
Educational level & & & \\
Primary & $25(62.5)$ & $9(42.9)$ & $2(16)$ \\
Secondary & $8(20.0)$ & $6(28.6)$ & $1(5.3$ \\
University & $7(17.5)$ & $6(28.6)$ & $44.4(3.8)$ \\
Age (years) & $45.5(6.7)$ & $46.4(8.5)$ & $7.5(2.0)$ \\
Undetectable VL (years) & $7.7(2.2)$ & $7.9(2.3)$ & $572.6(268.9)$ \\
Current CD4 (cells/mm $\left.{ }^{3}\right)$ & $676.1(291.2)$ & $769.6(284.4)$ & $122.9(140.6)$ \\
CD4 nadir (cells/mm $\left.{ }^{3}\right)$ & $181.1(178.4)$ & $233.7(190.0)$ & \\
\hline
\end{tabular}


scores in the HIV-infected patient group were significantly poorer in one of the anticipation speed tests (mean deviation time $(\mathrm{p}=0.03))$ and in one of the multiple reaction tests (mean reaction time $(\mathrm{p}=0.05)$ ). In contrast, in relation to bimanual visual-motor coordination, the HIV-infected patients obtained better results in all three tests $(p<0.01)$. There were no differences in the percentages of patients with scores below the recommended limits versus the general population, in any of the tests. On comparing the treatment subgroups (EFV versus PI), no differences were recorded in any of the study variables, and the differences with respect to the general population were the same as those described for the global group.

\section{Comments}

Driving is a complex activity requiring the integration of perception, attention and motor response. The ASDE DRIVER TEST N-845 [8] is a test battery with equipment homologated by the Spanish traffic authorities, designed to assess psychological and physical aptitude for obtaining the driving license in Spain. The battery includes tests relating to anticipation speed, bimanual visual-motor coordination, multiple reaction times, concentrated attention, and resistance to monotony. Aptitude is declared by the examiner after the global evaluation, though it is considered that the results should exceed percentile 10 in the reference tables. The ASDE DRIVER TEST N-845 has also been used to assess driving aptitude in other scenarios such as dementias [9] and residents after the duty shift [10].

The great interest in neurocognitive disorders among well controlled HIV-infected patients is not without controversy [11,12]. The results of our study support the existence of small differences in driving skill between these patients and the general population-with poorer results in some of the tests referred to anticipation speed and reaction time, but with better performance in bimanual coordination. Given the different age distribution with respect to the reference tables of the general population (data not shown), poorer performance could have greater importance. In contrast, the good results obtained in terms of bimanual coordination among the HIV-infected patients possibly could be attributed in part to widespread playing of videogames, which exercises skills of this kind.

On the other hand, EFV is well known to induce CNS alterations in the first weeks of treatment [13]. The effects over the long term are less clear, and in the present study we found no differences in test performance among the patients administered EFV versus those treated with PIs. Assignment to treatment based on supervising physician criterion could interfere with interpretation of these results, and factors such as a lower CD4 nadir among the patients treated with PIs theoretically would favor greater neurocognitive alterations in this group-a situation no observed in our study, however.

The strength of our study is that it involves a highly selected group of patients with few comorbidities, and who have received stable ART for long periods of time. In contrast, a limitation of the study is the fact that treatment assignment was based on physician criterion; as a result, there were baseline differences in relation to CD4 nadir and current count.

\section{Conclusion}

Assuming the limitations inherent to non-randomized, cross-sectional studies, we observed minor differences in test performance among the HIV-infected patients versus the data contained in the normality tables. These differences are of arguable clinical significance, and no differences were found in driving skills between the patients administered EFV and those receiving PI treatment on a chronic basis. Further studies are needed to evaluate the effects of EFV in the first weeks of treatment.

\section{REFERENCES}

[1] D. B. Clifford, S. Evans, Y. Yang, E. P. Acosta, K. Goodkin and K. Tashima, "Impact of Efavirenz on Neuropsychological Performance and Symptoms in HIV Infected Individuals,” Annals of Internal Medicine, Vol. 143, No. 10, 2005, pp. 714-721.

[2] T. Hawkins, C. Geist, B. Young, A. Giblin, R. C. Mercier, K. Thornton, et al., "Comparison of Neuropsychiatric Side Effects in an Observational Cohort of Efavirenz- and Protease Inhibitor-Treated Patients," HIV Clinical Trials, Vol. 6, 2005, pp. 187-196. doi:10.1310/92VR-FP24-J8GA-B49Q

[3] C. R. Fumaz, J. A. Munoz-Moreno, J. Moltó, E. Negredo, M. J. Ferrer, G. Sirera, et al., "Long-Term Neuropsychiatric Disorders on Efavirenz-Based Approaches: Quality of Life, Psychologic Issues, and Adherence,” Journal of Acquired Immune Deficiency Syndromes, Vol. 38, No. 5, 2005, pp. 560-565. doi:10.1097/01.qai.0000147523.41993.47

[4] R. K. Heaton, D. B. Clifford, D. R. Franklin Jr., S. P. Woods, C. Ake, et al., "HIV-Associated Neurocognitive Disorders Persist in the Era of Potent Antiretroviral Therapy CHARTER Study,” Neurology, Vol. 75, No. 23, 2010, pp. 2087-2096. doi:10.1212/WNL.0b013e318200d727

[5] S. Simioni, M. Cavassini, J. M. Annoni, A. R. Abraham, I. Bourquin, V. Schiffer, A. Calmy, J. P. Chave, E. Giacobini, B. Hirschel and R. A. Du Pasquier, "Cognitive Dysfunction in HIV Patients Despite Long-Standing Suppression of Viremia,” AIDS, Vol. 24, No. 9, 2010, pp. 1243-1250.

[6] T. D. Marcotte, T. Wolfson, T. J. Rosenthal, R. K. Heaton, 
R. Gonzalez, R. J. Ellis and I. Grant, "HIV Neurobehavioral Research Center Group A Multimodal Assessment of Driving Performance in HIV Infection," Neurology, Vol. 63, No. 8, 2004, pp. 1417-1422.

doi:10.1212/01.WNL.0000141920.33580.5D

[7] T. D. Marcotte, D. Lazzaretto, J. C. Scott, E. Roberts, S. P. Woods, S. Letendre and HNRC Group, "Visual Attention Deficits Are Associated with Driving Accidents in Cognitively-Impaired HIV-Infected Individuals,” Journal of Clinical and Experimental Neuropsychology, Vol. 28, No. 1, 2006, pp. 13-28. doi:10.1080/13803390490918048

[8] H. Monterde, "Real Decreto 772/97 ASDE Driver-Test N-845,” Examen Psicológico de Conductores en España con Equipos Normalizados, 2005.

[9] D. Badenes Guia, "Discriminación de la Valoración de la Capacidad de Conducción de Vehículos en Personas con Diagnóstico de Deterioro Cognitivo Ligero y Demencia en los Exámenes de Conducción Normalizados,” MAPFRE Medicina, Vol. 8, No. 2, 2007, pp. 98-107.

[10] C. Tornero, A. Ventura, M. Bourguet and I. Poquet,
"Evaluation of Driving Ability among Residents after the Duty Shift," Accident Analysis \& Prevention, Vol. 7, 2012, pp. 182-183. doi:10.1016/j.aap.2012.01.007

[11] M. Gisslén, W. Richard, R. W. Price and S. Nilsson III, "The Definition of HIV-Associated Neurocognitive Disorders: Are We Overestimating the Real Prevalence?” BMC Infectious Diseases, Vol. 11, 2011, pp. 356-360. doi:10.1186/1471-2334-11-356

[12] C. Torti, E. Foca, B. M. Cesana and F. X. Lescure, “Asymptomatic Neurocognitive Disorders Inpatients Infected by HIV: Fact or Fiction?” BMC Medicine, Vol. 9, 2011, pp. 138-142. doi:10.1186/1741-7015-9-138

[13] M. Nelson, H. J. Stellbrink, D. Podzamczer, D. Banhegyi, B. Gazzard, A. Hill and Y. van Delft, "A Comparison of Neuropsychiatric Adverse Events during 12 Weeks of Treatment with Etravirine and Efavirenz in a Treatment-Naive, HIV-1-Infected Population,” AIDS, Vol. 25, No. 3, 2011, pp. 335-340. doi:10.1097/QAD.0b013e3283416873 